\title{
Estadísticas y trascendencia de la violencia sexual en menores.
}

\author{
Statistics and significance of sexual violence in minors.
}

\section{L. Suárez Solá1 y F. J. González Delgado}

\section{RESUMEN}

Hemos recopilado 58 casos de denuncias por violencia sexual en menores durante 8 años (1993-200I). Nos planteamos estudiar algunas variables, como la frecuencia a lo largo de los años o meses para poder objetivar incrementos del número de incidencias en algún período como los vacacionales o de otro tipo. También estudiamos, entre otras variables, las horas de la exploración, el estado civil de las madres o el origen de la denuncia, observando que es la propia menor la que relata lo sucedido en un $67 \%$ de los casos originando la denuncia. El tiempo transcurrido entre los hechos y la denuncia es muy variable, observándose que es más corto cuando el agresor es desconocido o no tiene relación de parentesco con la menor. Cuando es un familiar o el propio progenitor suele tardar años en revelar los hechos, probablemente hasta que la niña descubra que esas actividades son incorrectas e injustas o que pueda defenderse de su agresor. En nuestra investigación el perpetrador es conocido en un $\mathbf{8 8 \%}$ de los casos, si bien se piensa que ocurre en todas las estratos sociales, pudiendo comprobar en este estudio una mayor frecuencia en el estatus social bajo, lo que podría interpretarse de muchas formas, bien porque ocurre menos en los niveles económicos altos, o porque en este estatus se denuncie menos. El estudio de nuestras observaciones son indicativos de que la marginalidad y la pobreza son frecuentes en este grupo de menores.

Palabras clave: violencia sexual, edades, agresores, origen de la denuncia, estadísticas.

\section{ABSTRACT}

We have compiled during eight years 58 cases on sexual violence allegations to minors. We have considered studying some variables like the incidence frequency throughout the years or months in order to assess if there was greater incidence in vacation periods, end-of-month, pay-check days, etc. We have also paid attention to other variables such as hours of the exploration; marital status of the mothers; origin of the allegation. For instance we have observed that it is the same minor who relates the allegation in $67 \%$ of the cases. The time elapsed between the event and the time a charge is presented varies widely, although it has been observed that it is shorter when the aggressor is not known or is not related to the minor. When it is a relative or the parent, it usually takes years for charges to be brought to justice, probably until the girl discovers that those activities are incorrect and unjust or she can defend herself against her aggressor. In our investigation the offender is known in $88 \%$ of the cases.

Although sexual abuse can happen in all social classes, in this study we could verify that it is more frequent in deprived, poorer classes. This can be interpreted in many ways. Either it happens less in wealthier classes, or they prefer to keep it quiet in order to avoid public scrutiny. Nevertheless the study shows that social outcast and poverty are frequent in this group of minors.

Key words: sexual violence, ages, aggressors, origin of the allegation, statistics.

Correspondencia: Palacio de Justicia, Plaza del Adelantado nº 3. La laguna (S/C de Tenerife). Teléfono: 629692452 FAX: 922 208073. Mail: msuasol@justiciaencanarias.org y frgondel@justiciaencanarias.org.

1 Doctor en Medicina y Cirugía, Especialista en Medicina Legal y Forense. Médico Forense Titular del Instituto de Medicina Legal de S/C. de Tenerife. 


\section{INTRODUCCIÓN:}

Para darnos cuenta de la magnitud del problema hemos realizado el cálculo de las personas implicadas en las acusaciones realizadas, teniendo en cuenta que además de las personas involucradas directamente (víctima y agresor) están los familiares o allegados más próximos. Esto lleva a unas ocho personas que pueden quedar afectadas por cada caso. Si esta cifra la multiplicamos por el número de víctimas estudiadas (58 casos) supondría casi medio millar de personas. Si pensamos que hay siete Médicos Forenses entre los Partidos Judiciales de Santa Cruz y La Laguna, siendo estos datos recogidos por solo dos de ellos, nos lleva a un importante número de personas involucradas (varios millares de personas) con un gran costo económico de todas las administraciones y de la sociedad en general.

Para aproximarnos a la realidad e importancia de este problema hemos recopilado los siguientes datos:

Los abusos sexuales constituyen el $9.6 \%$ de los maltratos en la infancia. El U. S. Departamento of Health \& Human Services [I] realizó un estudio en varios Estados ofreciendo el número de víctimas de abuso sexual por año, desde 1997 al 2001 , observando un descenso progresivo desde 1.7 a 1.2 (basado en el número de víctimas dividido por el número de niños en la población, multiplicado por 1000). Así, en el año 200 I de 51 estados, con una población de 72.941 .000 el número de víctimas fue de 86.830 equivalente a una proporción de I.2.

La gravedad del problema se viene estudiando desde hace tiempo, así Forward en 1993 estimaba que unos 60 millones de niños habían sufrido abuso sexual en la infancia en América [2]. Aproximadamente el $31 \%$ de las mujeres en las prisiones de los Estados Unidos sufrieron atropello sexual cuando fueron niñas [3], y el 95\% de las prostitutas adolescentes padecieron atentados sexuales [4]. También se ha estimado que las niñas con deficiencia psíquica son entre 4 y 10 veces más vulnerables que las que no la sufren [5].

La importancia de este tema no solo viene dada por su volumen sino por las lesiones, especialmente psíquicas, que pueden permanecer en el tiempo. Así, los efectos más frecuentes a largo plazo del abuso en niñas incluyen miedo, ansiedad, depresión, cólera, hostilidad, comportamiento sexual inadecuado, pobre autoestima, tendencia al abuso de sustancias y dificultad para relacionarse [6].

Los hallazgos clínicos en mujeres adultas, que sufrieron actividades sexuales ilegales en la infancia, se caracterizan por problemas en las relaciones interpersonales con una desconfianza subyacente. Por lo general, las mujeres que padecieron de incesto tienen una relación muy tensa con sus padres, que están marcadas por sentimientos de recelo, temor, ambivalencia, odio y traición. Estos sentimientos se extienden a todos los miembros de la familia [7]. Casi todas las víctimas experimentan un sentimiento de culpabilidad derivada del placer sexual [7]. Recordemos también que la sexualidad está relacionada no simplemente como una parte autolimitada de los genitales, o aspectos biológicos de la reproducción, sino que es más adecuado entenderla como un componente de la personalidad total que afecta al concepto de la identidad de la persona y autoestima [8]. Por ello, la victimización sexual puede interferir profundamente en el desarrollo y alterar las actitudes hacia sí misma, la sexualidad y la confianza en las relaciones durante los primeros años críticos de la vida [9]. Si la menor abusada no resuelve el trauma, la sexualidad puede convertirse en un área de conflicto de adulta $[8,9]$.

Las niñas que se sienten obligadas a mantener en secreto el atropello sexual sufren una mayor angustia psíquica que las que revelan el secreto y reciben asistencia y apoyo [ 10$]$. 
Las adolescentes con un historial de abuso sexual tienen más probabilidad de meterse en comportamientos sexuales de riesgo, según el Dr. Larry K. Brown et al., del Hospital de Rhode Island en Providence. Los antecedentes de atentado sexual se relacionan con una reducción en el control de los impulsos y un porcentaje más alto de enfermedades de transmisión sexual. Según el Dr. Brown, muchas de estas menores reexperimentan la ansiedad y el trauma durante años [I I].

La mayoría de los autores están de acuerdo en que la violencia sexual en la niñez se asocia con un riesgo mayor de posteriores desórdenes psiquiátricos [12]. También se ha sugerido que el abuso sexual puede favorecer que sean sexualmente activas a una edad más temprana y buscar novios de más edad, que a su vez pueden introducirlas en las drogas. Los problemas mentales fueron de 2,6 a 3,I veces más comunes entre las que sufrieron violencia con coito, y el riesgo de exceso de consumo de sustancias aumentó cuatro veces, según los resultados. Los factores familiares, educativos, de comportamiento de los padres, estatus financiero familiar y asistencia a la iglesia tuvieron poco impacto sobre la incidencia de las alteraciones de abuso de sustancias o psiquiátricos entre estas víctimas. De manera similar, la psicopatología de los padres no predice la asociación entre el abuso o agresión y la posterior psicopatología [13].

El historial de abuso sexual y físico entre las adolescentes aparece como un aumento del riesgo de padecer desórdenes en los comportamientos alimentarios, como el vómito autoinducido y el uso de laxantes para evitar el aumento de peso [14]. Otros autores como Waller et al. [15] han observado alteraciones en los hábitos alimenticios como la bulimia. Por otro lado, Teusch [16] ha descrito en una mujer un consumo excesivo de sustancias tóxicas secundario al atentado sexual sufrido en la niñez.

Wonderlich [17] sugiere que las niñas forzadas pueden experimentar niveles más elevados de angustia emocional, posiblemente ligado a los atentados sufridos, teniendo problemas para superarla. La limitación de la comida y quizás otras alteraciones en la conducta con los alimentos pueden reflejar los esfuerzos para afrontar sus experiencias [14].

Muchas menores que han estado sometidas a graves atropellos sexuales o físicos sufren a largo plazo trastornos mentales. También pueden verse invadidas por pesadillas y recurrencia de muchos síntomas similares a los sobrevivientes de una guerra $\mathrm{o}$, a la inversa, congelarse en una calma entumecida en situación de intenso estrés [1 8].

A nivel neuropatológico se han observado cambios en la morfología del hipocampo (área del cerebro que se ocupa de la memoria a largo plazo) sobre los que actúan hormonas que inundan el cerebro durante y después de un episodio estresante. Así Stein et al. [19], en un estudio en mujeres con antecedentes de abuso sexual en la niñez, objetivaron que tienen un hipocampo más pequeño en relación a las mujeres que no la han sufrido. Igualmente, Bremner y Narayan [20], refieren que hay evidencias de que el estrés afecta al hipocampo y que la capacidad de aprendizaje puede verse afectada. Si se confirma este descubrimiento, podría ser definido el perfil de una mujer que ha sufrido violencia sexual en la infancia y que hubiere padecido de stress postraumático.

\section{PLANTEAMIENTOS Y OBJETIVOS:}

La Medicina legal y Forense en la práctica diaria se relaciona con numerosas especialidades, teniendo también una función terapéutica, como la propia exploración de las menores, debiendo realizarse inmediatamente, antes incluso de la denuncia. Nuestro objetivo principal es mejorar nuestra pericia en este campo, e incentivar la investigación reflejando la importancia del problema, para que en definitiva se beneficie y ayude en su justa medida a estas víctimas. 


\section{MATERIAL Y MÉTODO:}

Hemos realizado un estudio de las denuncias por violencia sexual en niñas durante los años 1993 a 1998 en 5 de los II Juzgados de la ciudad de S/C. de Tenerife (municipios de Santa Cruz de Tenerife y El Rosario), y de 1998 a 200 I en 4 de los 6 Juzgados de La Laguna (municipios de La Laguna, Tegueste, Tacoronte y el Sauzal).

Durante este período de tiempo tuvimos 47 víctimas mujeres mayores de 18 años, 3 en hombres adultos, 20 en niños varones y 58 en niñas menores de 18 años. Nuestros resultados los centramos en estas 58 denuncias de niñas menores ( $<18$ años), recopilando los informes médico forenses con en los datos recogidos durante la primera exploración médico forense, realizados durante estos nueve años.

\section{RESULTADOS:}

Como variables estadísticas e indicadores de violencia sexual hemos elegido:

$>$ Edades.

Número de denuncias por año.

- Número de denuncias por meses.

$>$ Hora de la exploración.

$>$ Estado civil de la madre.

$>$ Origen de la denuncia.

$>$ Tiempo entre los hechos y la denuncia.

$>$ Estatus social de las menores.

Consentimiento de la víctima.

Agresor conocido o desconocido.

$>$ Parentesco del agresor.

- Acompañantes de la víctima en la exploración.

> Exploración psicológica.

Antecedentes psicológicos.

\section{Edades:}

La distribución es homogénea afectando a casi todas las edades siendo más frecuente, con ocho casos, a los 16 años, seguido de los 3, 9, 13 y 17 años con cinco casos. En las edades de I I, 12 y 14 años hubieron cuatro casos. A los 4 y 10 años tres casos y, cinco casos a los 2, 6, 7, I 5 y 18 años. Solo hubo un caso en una niña de un año y no hubo en nuestro estudio ningún caso a los ocho años (Gráfico I).

\section{Número de denuncias por año:}

En el estudio desde 1993 hasta 200 I pudimos observar una variación desigual, aunque con una tendencia ascendente. Por años, hay un pico en el año 2000 con 12 casos, seguido de los años I 995 con nueve casos, I 998 con ocho casos, 200 I con siete casos y los años I 993 y I 999 con seis casos. Los años en donde hubo menos denuncias fueron en 1996, 1997 y 1994 con 5, 3 y 2 casos respectivamente. (Gráfico 2).

\section{Número de denuncias por meses:}

La mayor frecuencia correspondió a los meses de septiembre, diciembre, junio y abril con $9,8,8$ y 6 casos respectivamente, seguido de febrero, mayo y julio con 5 casos. Los meses con 
menos denuncias fueron octubre, agosto, noviembre, marzo y enero con 3, 3, 2, 2 y 2 respectivamente (Gráfico 3).

\section{Hora de la exploración:}

Nosotros dividimos el horario del día en tres grandes grupos: por la mañana (de las 9 a las 15 horas), por la tarde (de las 16 a las 20 horas) y por la noche (de las 21 horas a las 8 de la mañana).

La distribución durante el día fue mayoritariamente por las mañanas con 35 casos, siendo por la tarde y por la noche prácticamente igual con 12 y I I casos respectivamente. Los picos mayores por la mañana fueron a las 1 I y 12 horas. Por la tarde fue más frecuente a las 18 horas, y por la noche no hay predominio marcado siendo la distribución horaria de forma homogénea. (Gráfico 4).

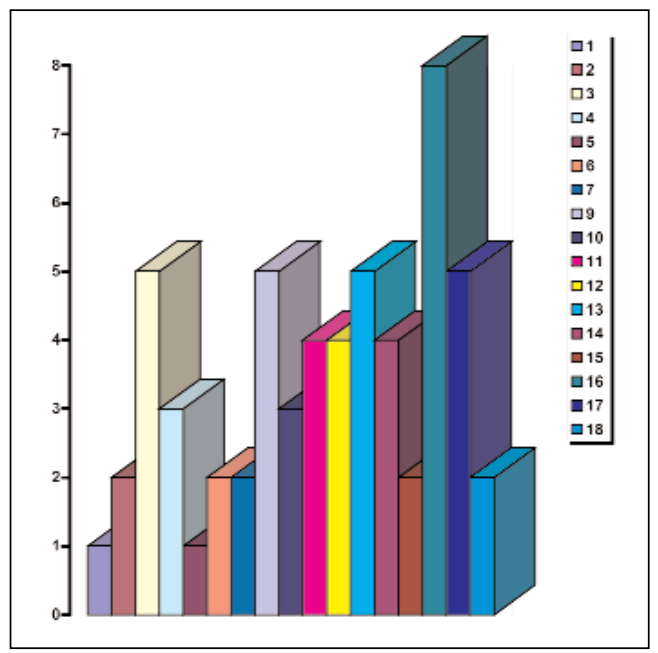

Gráfico 1.- Edades.

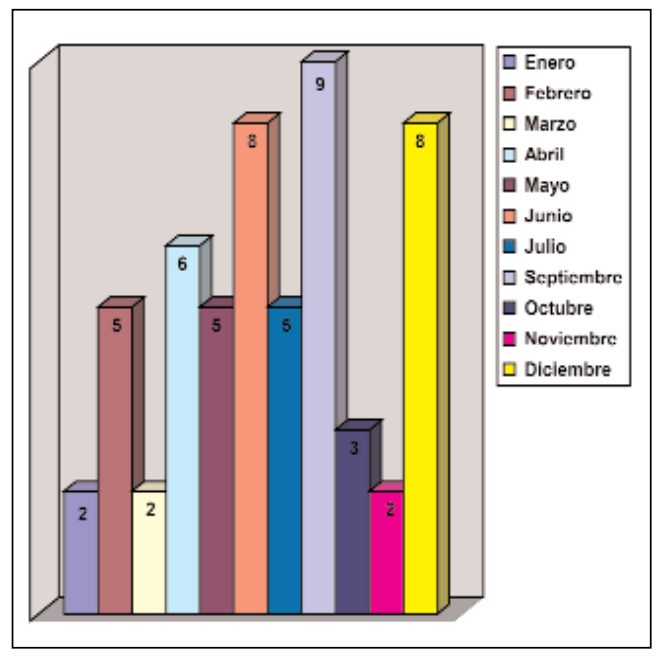

Gráfico 3.- Número de denuncias por meses.

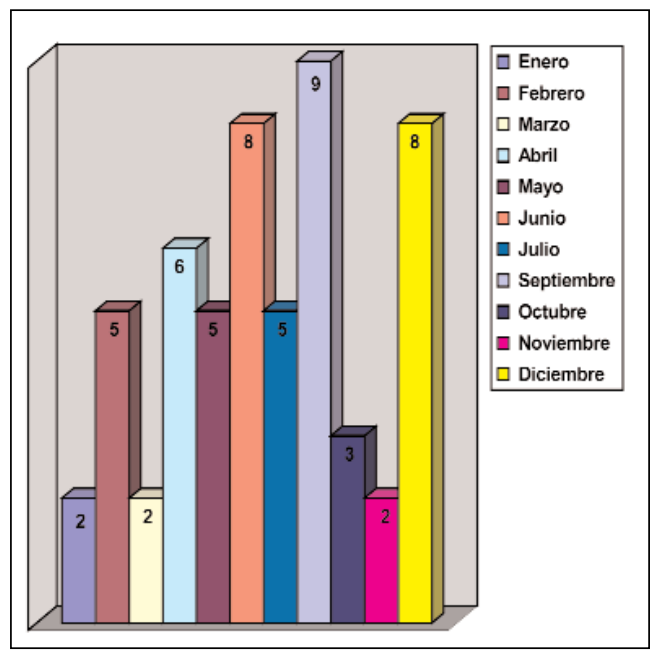

Gráfico 2.- Número de denuncias por año.

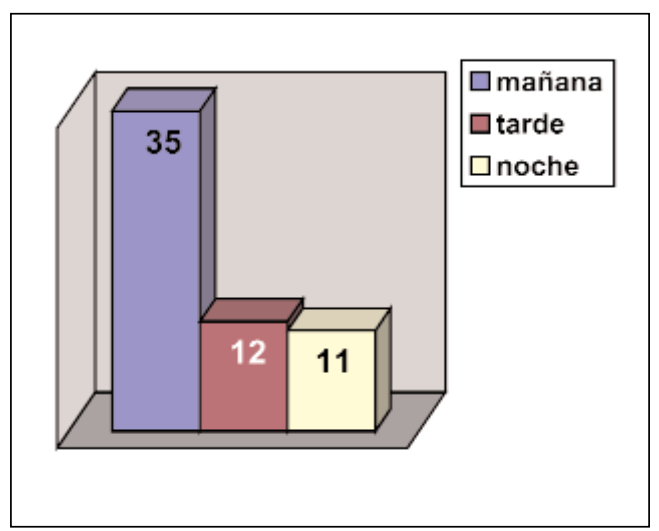

Gráfico 4.- Hora de la exploración. 


\section{Estado civil de la madre:}

La mayor parte de las madres de las niñas eran de estado civil casado con un total de 37 casos, seguidas de 10 casos separadas, 6 casos eran solteras, 4 divorciadas, y en un caso la madre de la menor había fallecido. (Gráfico 5).

\section{Origen de la denuncia:}

Es la propia niña la que en la mayoría de las ocasiones da la alarma para el inicio de la denuncia, siendo 39 casos del total de 58 estudiados los promovidos por la menor. La denuncia es iniciada por las madres en 13 casos, las cuidadoras en 3 casos, los abuelos con 2 casos y una madrina en una ocasión. (Gráfico 6).

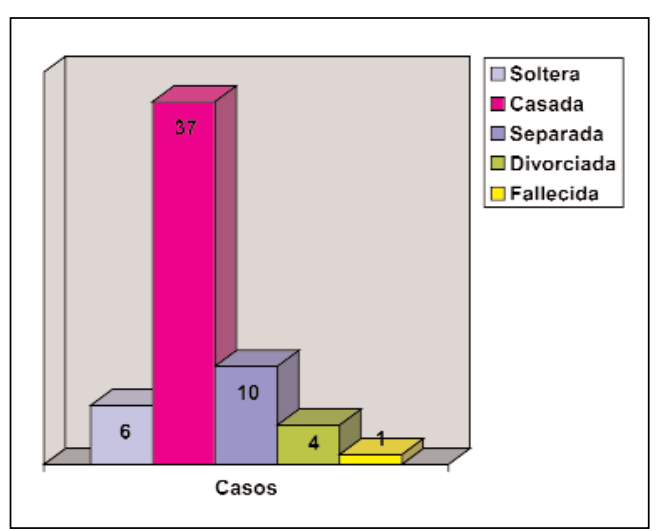

Gráfico 5.- Estado civil de la madre.

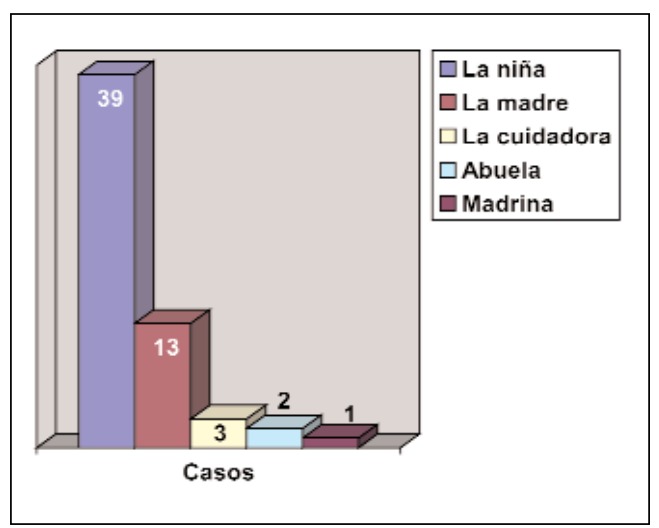

Gráfico 6.- Origen de la denuncia.

\section{Tiempo entre los hechos y la denuncia:}

Una variable muy importante desde el punto de vista médico legal es el tiempo que se tarda desde que ocurren los hechos hasta que se inicia la primera exploración forense, dada la importancia de la toma de muestras para encontrar la evidencia que le sirva al Juez para poder identificar al presunto agresor. En nuestro estudio hemos distinguido 5 grandes grupos de tiempo (horas, días, semanas, meses y años). Cuando han transcurrido varios días los hallazgos indiciarios se reducen considerablemente. En nuestros resultado pudimos observar que en el mayor número de casos pasaron varios días (2I casos), seguidos de 13 casos recientes (horas), en dos casos habían transcurrido semanas, en 9 casos pasaron varios meses antes de primera exploración, y en 13 casos transcurrieron mas de un año (Gráfico 7).

\section{Estatus social de las menores:}

El nivel socio-familiar de las menores fue mayoritariamente por debajo de la clase media, con un total de 39 casos, frente a 19 casos de nivel social medio. No se denunciaron agresiones o abusos a menores de estatus alto o muy alto. (Gráfico 8).

\section{Rechazo o consentimiento de la víctima:}

En nuestro estudio solo en dos casos afirmaron querer realizar los actos sexuales. Mayoritariamente, 56 de los 58 casos se negaron, no deseaban los abusos a los que fueron sometidas o dada su corta edad no podían entenderlos, estimándose que fueron obligadas a los mismos. (Gráfico 9). 


\section{Agresor:}

La gran mayoría de los casos el agresor correspondió a las personas del entorno de la víctima, siendo conocido por la misma en $5 \mathrm{I}$ de los 58 casos. Solo en 7 casos el agresor no era del entorno de la víctima. (Gráfico I0).

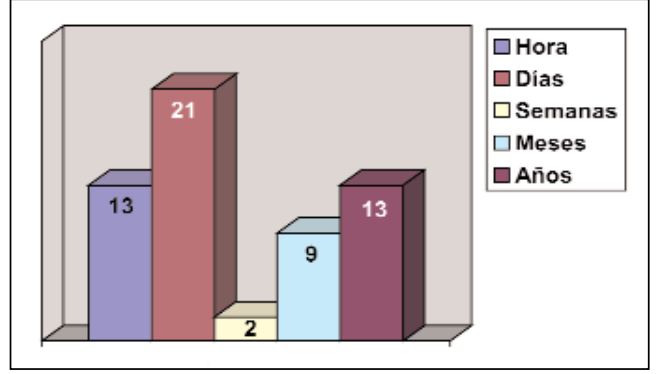

Gráfico 7.- Tiempo entre los hechos y la denuncia.

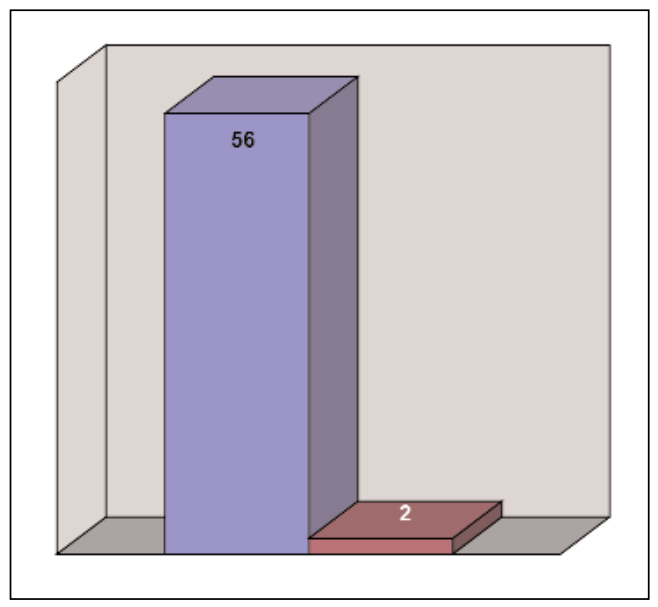

Gráfico 9.- Consentimiento de la víctima.

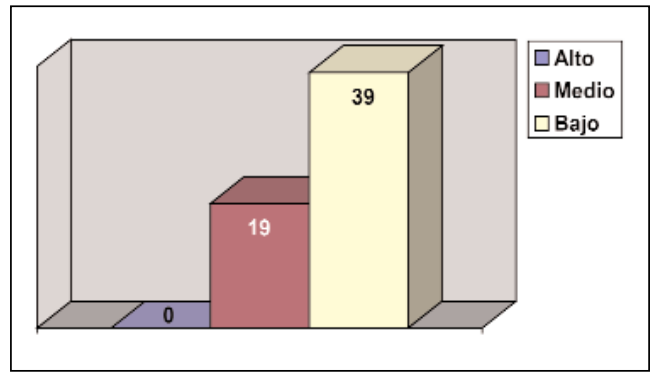

Gráfico 8.- Estatus social de las menores.

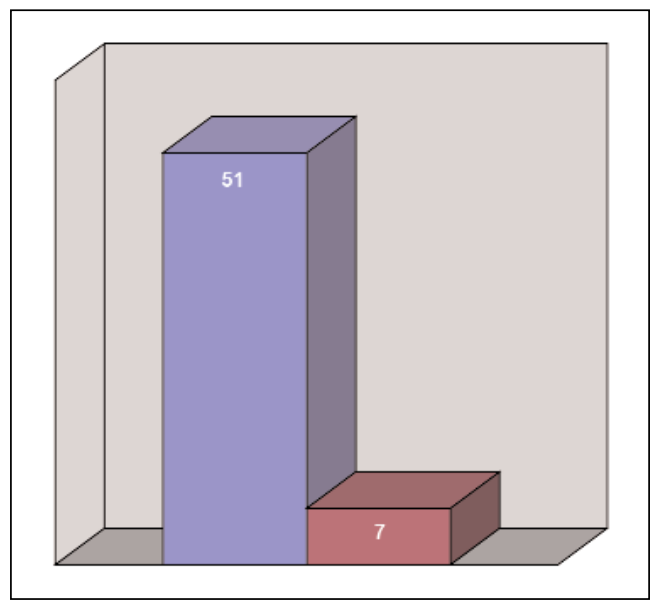

Gráfico 10.- Agresor conocido o desconocido.

\section{Parentesco del agresor:}

En los casos en que el autor es conocido existía mayoritariamente un parentesco paterno en 17 casos, en 3 casos fue un tío, en tres casos el agresor fue un abuelo y en dos ocasiones fue un cuñado. En 29 casos el agresor no tenía una relación de parentesco directa. (Gráfico I I).

\section{Acompañantes de la víctima en la exploración:}

La niña cuando acudió al examen forense lo hizo generalmente acompañada de su madre (42 casos), seguido con menor frecuencia por la cuidadora, hermanas o la policía, y de forma aislada por la madrina, tía, abuelo, amiga o acudió sola. (Gráfico |2).

\section{Exploración psicológica:}

El estado psíquico de las menores fue examinado de forma paralela con el físico. Atendiendo al estado de conciencia y comportamiento se observó que la mayoría (43 casos) no pre- 
sentaba alteraciones aparentes, 13 de las niñas presentaban un estado de inquietud o nerviosismo, una niña estaba adormilada y una era muy retraída (Gráfico I3). Por otro lado, atendiendo a los antecedentes psicológicos previos observamos que 45 de los 58 casos no presentaban trastornos mentales conocidos, en 10 casos existía un retraso mental en distintos grados, un caso tenía trastornos adaptativos, un caso con un intento de suicidio y depresión, y otro con ansiedad y agresividad (Gráfico |4).

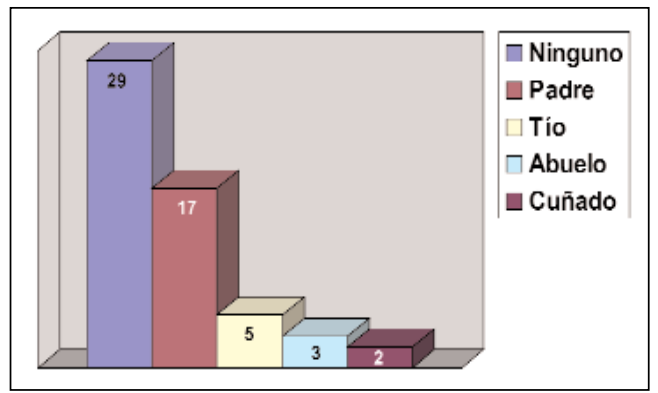

Gráfico 11.- Parentesco del agresor.

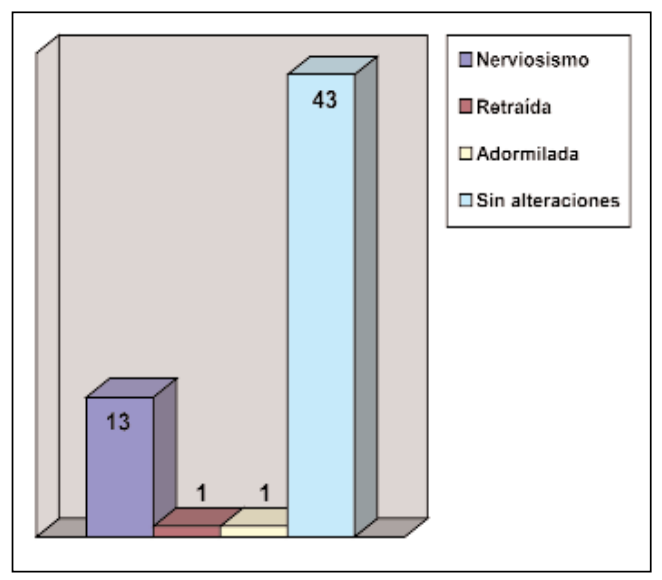

Gráfico 13.- Exploración psicológica.

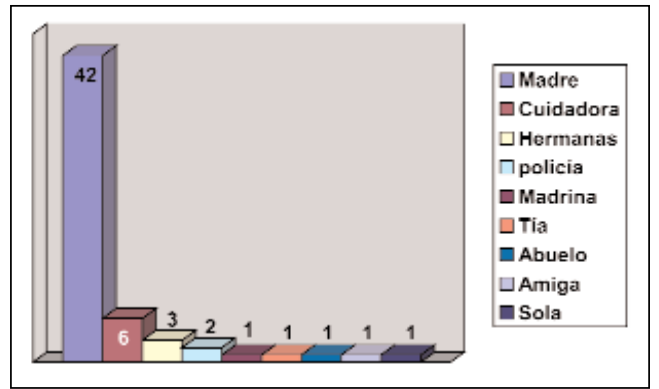

Gráfico 12.- Acompañante en la exploración.

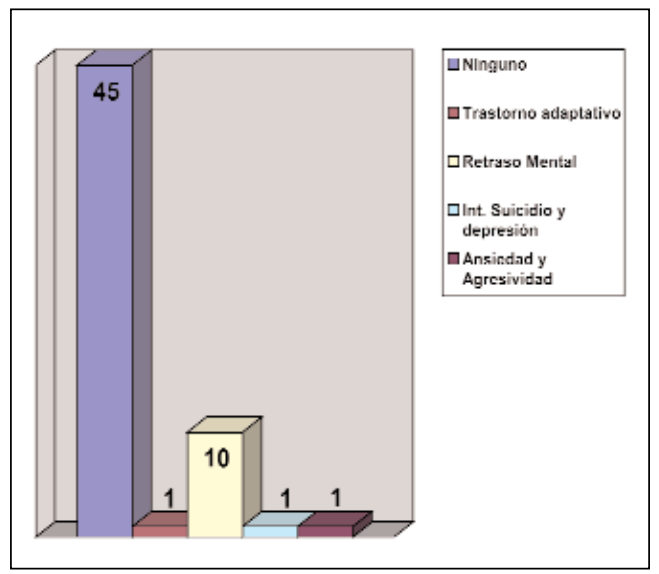

Gráfico 14.- Antecedentes psicológicos.

\section{DISCUSIÓN:}

\section{PROBLEMÁtICA de LAS ESTADíSTICAS.}

El primer punto de partida es definir el concepto de violencia sexual, ya que este varía según el país. Nosotros recogimos todos los casos de denuncia por violencia sexual, incluyendo con este término tanto las agresiones sexuales (con violencia o intimidación) como los abusos sexuales (sin violencia o intimidación) [2। ], es decir, los atentados contra la libertad sexual sin consentimiento (considerando no consentidos, en todo caso, a los que se ejecuten sobre menores de trece años, sobre personas que se hallen privadas de sentido o de cuyo trastorno mental se abusare) [2।]. Los atentados sexuales estudiados consistieron en tocamientos, intento de acceso carnal, acceso carnal por vía vaginal, anal o bucal, o introducción de objetos, ocurridos durante los años 1993 al 2001. 
Nuestro estudio se centró especialmente en el grupo de edades de 0 a 18 años por apartarse de la típica violación de la víctima adulta.

Los datos reales tienen una problemática muy específica que autores como Silverman señalan como "el resultante de un conflicto entre la baja detección de los casos reales y la sobreinformación de los casos que aparecen individualmente" [22].

En nuestro país, apenas hemos encontrado estudios estadísticos completos y pocos trabajos de investigación cuando se trata de víctimas sexuales en menores. Las estadísticas de los distintos países utilizan la definición de la violencia sexual adaptada a sus legislaciones respectivas. Sin embargo, los investigadores independientes utilizan definiciones diversas, por ejemplo, violencia sexual leve o moderada. En España el Instituto Nacional de Estadística engloba a todos los delitos contra la libertad sexual sin discriminar los distintos tipos ni las edades.

Nuestro trabajo ha recopilado datos retrospectivos, con los problemas de información que conlleva, y hemos realizado un estudio descriptivo y simplificado de los datos [23]. Esto puede llevar a diferencias con otras estadísticas haciendo difícil un estudio comparativo dado que no parten todas de las mismas variables, conceptos y definiciones. En general, en los Estados Unidos los abusos sexuales en menores forman una parte de los maltratos en los niños ocupando un $10 \%$ aproximadamente de los mismos [24].

Nosotros nos hemos centrado en los abusos sexuales y en los intentos o agresiones sexuales, siempre que haya intervenido el médico forense en la exploración física genital de las niñas, excluyendo los varones y los delitos de exhibicionismo o la utilización de menores para pornografía al no ser práctica habitual en la actividad forense. Debemos descartar también que hay diferencias sustanciales entre las niñas más pequeñas de las adolescentes dado que en las primeras no están desarrolladas físicamente ni preparadas mentalmente para comprender los actos a los que se las someten.

Otro factor importante que puede influir en los resultados estadísticos es la objetividad en la recogida de datos, que pueden ayudar a comprender mejor este tipo de delitos tales como el estado civil de las madres o separación de los padres y problemas socioeconómicos entre otros.

Todo ello hace necesario el uso de protocolos normalizados y consensuados en las entrevistas y exploraciones realizadas tanto por forenses y psicólogos, así como por otros profesionales como los ginecólogos y pediatras que evaluarían la evolución del desarrollo físico y psíquico de las menores hasta los 18 años. También es conveniente escoger las variables que puedan resultar de utilidad desde el punto de vista pericial, terapéutico y preventivo que nos hagan conocer mejor estos delitos y poder actuar de la forma más eficaz sobre los mismos, siendo esta la intención de nuestro trabajo, preservando siempre la identidad de las víctimas.

Debemos añadir como reflexión final, que para conocer la realidad estadística de estos casos tendríamos que recoger los datos tanto físicos como psicológicos en todas las exploraciones, añadiendo los factores familiares y sociales que se estimen de interés. Teniendo en cuenta que es imprescindible utilizar la misma definición de violencia sexual, realizar protocolos consensuados y diseñar una base de datos y un sistema informático que estudiara las estadísticas en cada Instituto de Medicina Legal y posteriormente del conjunto global, consiguiendo evaluar la realidad del país, pudiendo hacer comparaciones en las distintas ciudades y autonomías, y establecer las influencias sociales, familiares, económicas, etc que pudieran influir en su incidencia, en la terapéutica y sus posibles soluciones.

Con la puesta en marcha de los diferentes Institutos de Medicina Legal se crearán las Comisiones de Investigación en colaboración de las Cátedras de Medicina legal, previsto en el Reglamento de los distintos institutos [25, 26], que son el fundamento de medios personales y materiales para realizar la investigación de estos campos. 
Este trabajo pretende dar a conocer la importancia de estos delitos, estableciendo la necesidad de establecer estadísticas fiables que puedan ser origen de futuras investigaciones en este tema. Recordemos que la finalidad de los Institutos es la formación de los médicos forenses y la capacitación de expertos en distintas áreas dentro de las que ocuparía especial relevancia la sexología forense en menores. Nuestras perspectivas futuras en la investigación de este campo estarán integradas con la puesta reciente en funcionamiento del Instituto de Medicina Legal de S/C. de Tenerife [26], y que esperamos se vayan generalizando.

\section{MotivaCiOnES DE "NO" DENUNCIAR.}

Esta motivación negativa viene referida por López [27] a una falta de conocimientos sobre el abuso, como son, la obligación de acusar, la importancia sobre sus efectos en la niña, el procedimiento a seguir, una inadecuada percepción del rol profesional y del deber cívico pensando en que no es cosa suya; atribución del coste profesional negativo (pérdida de tiempo, conflicto con la familia, reputación, ... y y en las consecuencias negativas para el agresor e ineficacia del sistema. En nuestra experiencia, a las causas que pudieran justificar esta falta de acusaciones se le añaden, el miedo a delatar y el temor al examen físico, que es considerado como un acto desagradable y no como una exploración médica normal. Otros razonamientos que encontramos son: el terror a que existan represalias por parte del agresor, el recelo a que no la crean o el deseo de mantener el anonimato, especialmente en los casos en que el perpetrador es un familiar. En estos casos las dificultades se incrementan al existir dependencia económica, lazos afectivos o de otra índole.

Indudablemente, hay que ser valiente para revelar los hechos delictivos, pero recordemos que el futuro desarrollo y la maduración de muchas menores podría afectarse si no se denuncia, como lo reflejan las investigaciones neurobiológicas ya señaladas en estudios cerebrales. Aunque el sistema tenga muchos defectos, se debe acusar, debemos investigar y estudiar para mejorarlo, pero nunca ocultarlo porque el número de víctimas irá en aumento.

El abuso sexual no es un "juego sexual", como algunos lo pueden considerar, así, no es lo mismo que de niños durante el crecimiento se miren o toquen los genitales unos a otros, debido a una mutua curiosidad o descubrimiento, sin coerción o intrusión del cuerpo, que estaría considerado como una conducta normal. Sin embargo, cuando un niño trata de obligar a una niña de 3 años de edad a un coito exhiben un comportamiento sexual inapropiado pudiendo estar reaccionando a su propia victimización.

Otro factor que se plantea para aumentar las denuncias es asegurar el anonimato, especialmente en el ámbito judicial, debido a que engendraría una mayor seguridad en las víctimas que, sumado a su protección, animaría a realizar estas revelaciones alejando los posibles miedos a represalias que rondan la mente de las menores.

\section{VALORACIÓN DE LOS RESULTADOS POR EDADES.}

Como hemos observado en nuestro trabajo la edad más frecuente suele ser entre los 13 18 años, siendo los 16, en plena adolescencia, lo más frecuente. Esto pudiera estar debido a que a esta edad ya existe un desarrollo de los caracteres sexuales secundarios y sin embargo persiste la inmadurez psicológica y dependencia familiar. Por otro lado, también hemos podido observar que, aunque los hechos hayan datado de tiempo atrás, a la edad de la adolescencia salen a relucir. Esto puede explicarse por el estado de madurez psíquica y rebeldía propia de la edad que hace comprender y criticar los hechos que no comprendían en edades más precoces. No obstante, se ha podido constatar que a pesar de la mayor frecuencia en estas edades existen casos en todas las edades de la infancia. En atención a las lesiones genitales en las menores hay que tener en cuenta que 
el coito es anatómicamente imposible por debajo de los 6 años debido a que el ángulo subpúbico es muy agudo. Entre los 6 y los II años puede producir la rotura del periné o incluso el tabique rectovaginal, siendo a partir de los II años las lesiones genitales ligeras limitándose casi exclusivamente a la rotura del himen [28]. En nuestro estudio [30] pudimos observar que la mayoría de los casos denunciados las lesiones físicas fueron mínimas debido, entre otros factores, a que se limitaron en ocasiones a tocamientos.

Relacionando nuestros datos podemos señalar, con las debidas precauciones, que las estadísticas en general, no discriminan por edades, en ocasiones, añaden todos los tipos de maltratos en menores y no cada tipo particular de violencia sexual, y por otro lado, no siempre se basan en el mismo concepto de definición de violencia sexual. En cuanto a la edades y años siguiendo las estadísticas del [I] U.S. Department of health and human services, reflejan de 0-3 años un 27, 7\%, de 4-7 años un 24, I \% de 8- II años un 22,8\%, de I2- I 5 años el 19,5\%, de 16 - 17 años el 5,4\% y a los 18 el $0,2 \%$. En los casos de violencia sexual recogidos de los datos estadísticos de Bureau of Justice Statistica (BJS) [24] del año 2000 señalan que el 67\% de las víctimas juveniles están alrededor de los 18 años, el 34\% de las agresiones sexuales están alrededor de los 12 años, y I de cada 7 víctimas de agresiones sexuales está debajo de los seis años. Hay una importante diferencia entre las que incluyen todos los malos tratos o las que especifican los relativos a la violencia sexual. Nuestros resultados coinciden con estos últimos datos que estiman que la mayoría se encuentra entre los 12 y los 18 años.

Un dato a destacar es que sobre los seis años en adelante las menores empiezan a relatar los hechos que le han sucedido, aunque esto se produce en ocasiones años después de sufridas las agresiones.

\section{Relación temporal AÑos-Meses-Horas.}

Otro dato interesante es la evolución de las denuncias con los años ya descritos en la introducción, donde se aprecia una reducción de estos delitos en estos últimos años coincidiendo con los datos del Instituto Nacional de Estadística de España en los años 2000 y 200 I. Sin embargo la proporción de nuestros resultados es muy superior dado que estudiamos todos los casos que llegan al Juzgado de Guardia, muchos de ellos se archivan por falta de pruebas, falta de autor u otras circunstancias, algunos después de la exploración ni siquiera se denunciaron, en cambio, los datos que reflejan el INE son solo los casos enjuiciados en los Juzgados de lo Penal y Audiencias Provinciales.

En cuanto a los meses suelen coincidir con el fin o principio del año escolar o con el inicio o el término de las vacaciones. Pensamos que esta relación con el período vacacional pudiera estar relacionado con la mayor permanencia en el hogar. También debemos señalar que generalmente dichos actos tienen lugar cuando no está presente la madre $u$ otros testigos.

\section{RETRASO DE LA DENUNCIA, EN RELACIÓN CON LAS EDADES Y PARENTESCO DE LOS AGRESORES.}

Si bien es la menor la que cuenta lo sucedido en un 67\%, el tiempo entre los hechos y la denuncia es muy variable, objetivándose la misma proporción entre las que acuden inmediatamente y las que tardan años. El retraso depende en muchas ocasiones de que el agresor sea conocido o que tenga relación de parentesco con la menor, que cree dudas sobre si le merece la pena denunciar los hechos, y en otras ocasiones, el retraso se produce hasta que la niña alcanza determinada edad y descubre que esas actividades son incorrectas o injustas y pueda defenderse de su agresor. En nuestra investigación el perpetrador es conocido en un $88 \%$ de los casos, siendo en un $50 \%$ un familiar, y su propio padre en un $29 \%$. No coincidiendo con otras estadísticas en que las víctimas pre- 
escolares de estos abusos sexuales fue intrafamiliar en un 72.5\%. En los escolares por encima de los seis años el abuso sexual mayoritario es el extrafamiliar, representado por el $73 \%$ y la duración del abuso sexual intrafamiliar fue prolongada [22], coincidiendo con nosotros en este último punto.

\section{VARIABLES RELACIONADAS CON EL RETRASO EN LA DENUNCIA.}

Analizando las variables que hemos recopilado podemos observar relaciones y diferencias entre ellas. Un caso especialmente significativo es la relación entre la edad y el tiempo de denuncia. A este respecto observamos que el grupo de las 14 niñas más pequeñas (entre 0-6 años) acudieron con bastante prontitud, generalmente durante horas y como máximo pocos días. En el grupo mediano entre 7-12 años, con 18 casos, solo tres acudieron rápidamente, el resto tardó días, semanas e incluso 7 tardaron más de un año en denunciar. El tercer grupo, de 13-18, con 26 niñas, se distribuyó de la siguiente forma: 7 acudieron en horas, 6 en días, 7 en semanas y 6 en más de un año. De este estudio estadístico se puede observar que las niñas más pequeñas son las que primero son llevadas al médico lo que sugiere la inquietud o preocupación básicamente solo de la madre, para salir de dudas. Observando en nuestra experiencia que tras el resultado negativo de la exploración, al tratarse de tocamientos en la mayoría de estos casos, la madre queda más tranquila y en un número apreciable de casos no denuncia los hechos o no quiere continuar. Por otro lado, observamos también que las del tercer grupo acuden en una proporción importante también de forma temprana al reconocimiento, pudiendo explicarse esta circunstancia a su mayor independencia de sus padres y mayor estado de madurez ( I 3- I 8 años) que le permiten comprender con mayor claridad los hechos y tomar por sí mismas la decisión de denunciar. En cambio en las niñas de edad intermedia (entre los 7 y 12 años) suele existir una amenaza por parte del agresor y dado que presentan todavía gran inmadurez y dependencia familiar se les crea una sensación de inseguridad y de culpa con miedo a que si se conocen los hechos va a repercutir en la ruptura o separación de sus padres, lo que hace que en algunas ocasiones se prolonguen los abusos, se produzca un fracaso escolar y la denuncia tarde en producirse. Así se ha podido observar que de los I I casos de amenazas verbales 7 tardaron años en denunciar.

Otra observación a la que hemos podido llegar es que cuando las amenazas han sido físicas (generalmente mayores de 16 años) la denuncia ha sido muy precoz, en cambio cuando la amenaza es de tipo verbal se ha tardado más en la denuncia.

Un dato que nos ha llamado la atención ha sido el hecho de que las niñas que más tardaron en denunciar los hechos (durante años), un número considerable (8 de las 10) sufrían un retraso mental. Esta observación nos lleva a relacionar los trastornos mentales con el retraso en la denuncia, es decir, presentarían una mayor dificultad en dar a conocer los abusos sufridos siendo con ello más vulnerables a este tipo de delitos.

\section{NIVEL SOCIAL Y ESTADO CIVIL DE LAS MADRES.}

Si bien se piensa popularmente que ocurre en todas las estratos sociales, en este estudio hemos podido observar una mayor frecuencia en el estatus social bajo, seguido del status medio. Esto se puede interpretar de muchas formas, bien porque no ocurre en los niveles económicos altos o porque se denuncia menos en estos niveles. No obstante, los datos objetivos son indicativos de que la marginalidad y la pobreza junto con el alcoholismo y la drogadicción son factores habituales en nuestros agresores. Por otro lado, en el estudio realizado no hay una relación clara y diferencial con respecto al estado civil de las madres, que puede hacer pensar que el mismo no es determinante, estimándose, no obstante, que habría que profundizar en estudios más numerosos para poder sacar conclusiones a este respecto. 


\section{RECHAZO DE LAS MENORES O CONSENTIMIENTO Y LÍNEAS GENERALES DE PREVENCIÓN.}

Como hemos visto las menores suelen rechazar la realización de la actividad sexual impuesta por el agresor. En todo caso, el consentimiento en menores de 13 años no sería válido nunca [2 I ]. Indicar que los pocos casos en los que admitió la relación fue, entre otros motivos, para agradar a la persona mayor, creyendo que era un "juego", considerando que eran normales los actos impúdicos a los se le sometían.

Las niñas abusadas sexualmente y su familia necesitan de inmediato evaluación profesional y tratamiento. Los especialistas pueden ayudar a la menor violentada a recuperar su autoestima, a saber sobrellevar los sentimientos de culpa del abuso, y empezar a vencer el trauma. Tales tratamientos pueden ayudar a reducir el riesgo de que la niña desarrolle diversos problemas cuando sea adulta.

En nuestra experiencia tras la denuncia y posterior instrucción y juicio, en su caso, perdemos total contacto con las víctimas, no asegurándose un seguimiento o vigilancia preventivo futuro por ninguna institución. Por ello, pensamos que la apertura de la denuncia debiera obligar a un equipo multidisciplinar a seguir la supervisión de esas menores hasta la edad adulta.

Se precisa un equipo único que investigue, estudie y dirija a todos los profesionales implicados. El estudio debe ser realizado por un equipo con conocimientos específicos para que sea efectivo y se obtenga una información válida [29]. Debemos recordar que la exploración física en las condiciones adecuadas ayuda psicológicamente tanto a la presunta víctima como a los familiares próximos, refuerza el diagnóstico y puede reducir las secuelas futuras [30].

Kuehnle [3।] hace hincapié en la importancia de la cualificación e independencia que deben tener estos peritos forenses. El examen médico tiene un enorme potencial tranquilizador de la normalidad e integridad física. Esto puede ayudar a restituir la autoestima e iniciar el proceso de recuperación, un efecto llamado "efecto terapéutico primario" [32].

Creemos que algunos consejos pueden ser útiles, como decirle a la niña "si alguien trata de tocarte tu cuerpo y hacer cosas para que te sientas divertida, di NO a la persona y cuéntalo enseguida.". Hay que enseñarles a los niños que el respeto no quiere decir una obediencia ciega en todo a los adultos y a la autoridad, por ejemplo, no debería aconsejarse "siempre haz todo lo que el profesor o lo que la canguro te diga". Debemos animar los programas de prevención profesional en los colegios.

\section{CONCLUSIONES:}

I. La violencia sexual en las niñas es más frecuente entre los 13 a 18 años. La edad más frecuente son los 16 años, habiendo también picos significativos a los 5 y 9 años.

2. Hay un promedio de seis casos por año, con un mínimo de dos y un máximo de doce por cada dos forenses.

3. Los meses con más denuncias corresponden a septiembre, junio y diciembre, con un mínimo de dos casos por mes y un máximo de nueve casos.

4. La exploración se realizó en el $60 \%$ de los casos por la mañana y el resto, en casi igual proporción, por la tarde y noche.

5. El estado civil de las madres más frecuente fue de casada.

6. El origen de la denuncia en un $67 \%$ provenía de la propia menor.

7. El tiempo transcurrido, desde que ocurrieron los hechos hasta la primera exploración, suele ser días y semanas, y luego horas y años en igual proporción.

8. El estatus social bajo es el más frecuente en estas menores. 


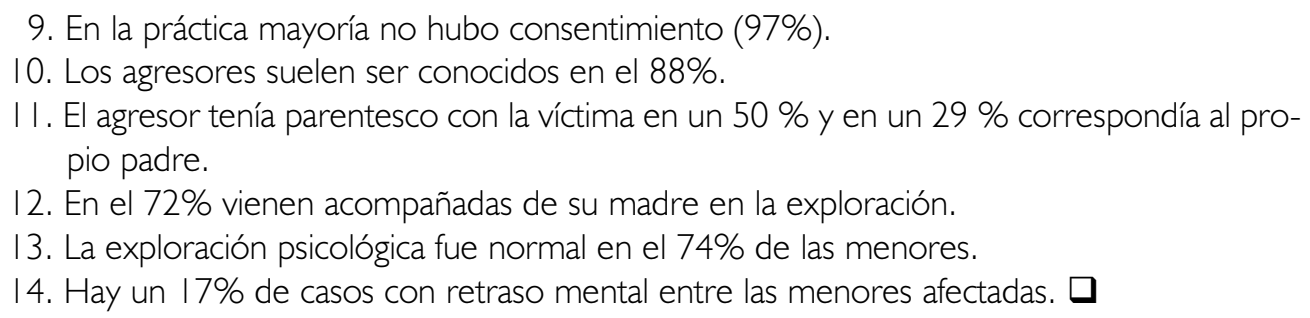

\section{BIBLIOGRAFÍA:}

I. U.S. Department of Health \& Human Services Child Maltreatment $200 I$ Administration for Children and Families. Administration on Children, Youth and Families. Children's Bureau 2001.

2. Forward. United Status Advisory Borrad on Child Abuse Statistics, 1993.

3. United Status Department Of Justice. About a third of women in prison say they were abused as children, 1991.

4. CCPCA. Connecticut Center for Prevention of Child Abuse. "http://www.ezboard.com". USA, 1992.

5. Centro Nacional de Recursos del Niño. Abuso Sexual 1992.

6. Brown WA \& finkelhor D. Impact of Child Sexual Abuse: a Review of the Research. Psychology Bulletin ,1986; 99(I):66-77.

7. Tsai $M \&$ Wagner $N$. Therapy groups for woman sexually molested as children. Arch. of Sexual Behaviorn, 1978; 7:417-427.

8. Whitlock K, \& Gillman R. Sexuality: a neglected component of child sexual abuse education and training. Child Welfare, 1989; $68,317-29$.

9. Courtois CA \& Watts DL. Counseling adult women who experienced incest in childhood or adolescent. The Personnel and Guidance Journal, 1982; 275-279.

10. Finkelhor D \& Browne A. Impact of child sexual abuse: a review of the research. Psychological Bulletin, 1986; 99, 66-77.

II. Larry K \& Brown MD, et al. Teens who have sexually abused are there times as likely to be inconsistent in their condom use. American Journal of Psychiatry, 2000; 157:1413-1415.

12. Bulik CM, Prescott CA, Kendler KS, Features of childhood sexual abuse and the development of psychiatric and substance use disorders. Br J Psychiatry 2001; 179:444-9

13. Kenneth S. Kendler MD, et al. Young girls who have been raped are three times more likely to suffer from psychiatric disorders and substance abuse problems as adults. Medical College of Virginia Commonwealth University, Archives of General Psychiatry, 2000; 57:953-959.

14. Dianne Neumark-Sztainer, et al. Sexual abuse increases the risk of succumbing to eating disorders among teen-age survivors, both male and female. University of Minneapolis, International Journal of Eating Disorders, 2000; 28:249-258 .

15. Waller G, Meyer C, Ohanian V, Elliott P, Dickson C, Sellings J. The psychopathology of bulimic women who report childhood sexual abuse: the mediating role of core beliefs. J. Nerv Ment dis 2001; 189:700-8.

16. Teush R. Substance abuse as a symptom of childhood sexual abuse. Psychiatry ser 200I; $52: 1530-2$.
17. Wonderlich SA, Wilsnack RW, Wilsnack SC, Harris TR. Childhood sexual abuse and bulimic behaviour in a nationally representative sample. Am J Public Health, 1996; 86:1082-1086.

18. Banyard VL, Williams LM, Siegel JA. The long-term mental Health consequences of child sexual abuse: an exploratory study of the impact of multiple traumas in a sample of women. J. Trauma Stress, 2001; 14: 697-715.

19. Stein MB, Koverola C, Hanna C, Torchia MG, Mcclarty B (1997). Hippocampal volume in women victimized by childhood sexual abuse. Psychol Med,I997; 27(4):951-9.

20. Bremner JD \& Narayan M. The effects of stress on memory and the hippocampus throughout the life cycle: implications for childhood development and aging. Dev. Psychopathol. Fall,1998;10(4):87I-85.

21. Ley Orgánica II/I999 que modifica los capítulos I y II del Título VIII del Libro II del Código Penal.

22. Cobo Plana JA. Asistencia integral a las mujeres víctimas de agresión sexual Editorial MASSON, SA. 1998 ppl-4.

23. Carrasco J.L. El Método Estadístico en la Investigación Médica 3

Ed. Ciencia, S.A. 1986 pp $18-35$

24. Bureau Of Justice Statistics (BIS).

25. R.D. 296/1996. Que desarrolla los Institutos de Medicina Legal en España.

26. R.D.198/2002, de 20 de diciembre que crea el Instituto de Medicina Legal en Santa Cruz de Tenerife, entrando en vigor el 25 de marzo de 2003.

27. López F. Prevención de los abusos sexuales de menores y educación sexual. Amarú, 1995; II4.

28. Gisbert Calabuig JA. Gisbert grifo MS. Delitos contra la libertad sexual. En Gisbert Calabuig JA. Medicina Legal y Toxicología $5^{a}$ ed. Masson. 1998 pp 495-514.

29. Carnes CN, Nelson-Gardell D, Wilson C, Orgassa UC. Extended forensic evaluation when sexual abuse is suspected: a multisided field study. Child Maltreat. 2001;6(3):230-42.

30. Suárez sola ML y González Delgado FJ. Importancia de la exploración médico forense en las agresiones sexuales a menores. Cuadernos de Medicina Forense. 2003:(31):37-45.

3I. Kuehnle K. Ethics and the forensic expert: a case study of child custody involving allegations of child sexual abuse. Ethics Behave, 1998; 8(I):I-18.

32. Furnis T, Bingley-Miller L, Bentovim A. Therapeutic approach to sexual abuse. Arch Dis Child.1984; 59:865-870. 